\title{
From the Fermi glass towards the Mott insulator in one dimension: Delocalization and strongly enhanced persistent currents
}

\author{
Peter Schmitteckert ${ }^{1,2}$, Rodolfo A. Jalabert ${ }^{1}$, Dietmar Weinmann ${ }^{3}$ and Jean-Louis Pichard ${ }^{2}$ \\ 1: Institut de Physique et Chimie des Matériaux de Strasbourg, 23 rue du Loess, 67037 Strasbourg cedex, France \\ 2: CEA, Service de Physique de l'Etat Condensé, Centre d'Etudes de Saclay, F-91191 Gif-sur-Yvette, France \\ 3: Institut für Physik, Universität Augsburg, 86135 Augsburg, Germany
}

\begin{abstract}
When a system of spinless fermions in a disordered mesoscopic ring becomes instable between the inhomogeneous configuration driven by the random potential (Anderson insulator) and the homogeneous one driven by repulsive interactions (Mott insulator), the persistent current can be enhanced by orders of magnitude. This is illustrated by a study of the change of the ground state energy under twisted boundary conditions using the density matrix renormalization group algorithm.
\end{abstract}

PACS 05.45+b, 72.15 Rn, $71.30+\mathrm{h}, 73.20 \mathrm{Dx}$

For a fixed kinetic energy $(t=1)$, the interplay between the disorder $(W)$ and a repulsive interaction $(U)$ is one of the central problems of mesoscopic physics [1]. For instance, its understanding appears as a necessary step towards explaining why the measured persistent currents [2] $J$ in mesoscopic rings are about two orders of magnitude larger than the value predicted by the noninteracting theory [3]. This discrepancy has prompted to study the role of the interaction using perturbation theory [4], assuming a Luttinger liquid and using renormalization group (5] for the disorder, or considering the limit where the rotational invariance of the interacting system is broken by the disorder [6]. On the other hand, exact numerical calculations on small $1 d$ models of spinless fermions have led to the conclusion that repulsive interactions cannot significantly [7] enhance $J$, being on the contrary rather detrimental $[8]$ to the expected effect. It was then argued that this is an artifact of spinless $1 d$ models, and that the full problem with spin does exhibit [5] the expected enhancement. We show in this Letter that even without spin, repulsive interactions can give rise to a large enhancement associated with a charge reorganization of the ground state. The interaction for which the ground state is reorganized fluctuates from sample to sample. Thus, the effect could not be seen in the previous studies of the ensemble averages, but it becomes very striking if one studies the orbital response of individual mesoscopic samples as a function of $U$.

Another motivation to re-examine the ground state of one dimensional spinless fermions stems from Shepelyansky's proposal concerning the role of interactions in an Anderson insulator [9]: two interacting particles (TIP) can be localized on a length $L_{2}$ much larger than the one-particle localization length $L_{1}$. Large ratios $L_{2} / L_{1}$ characterize the states in the bulk of the TIP-spectrum. However, a TIP ground state built from two one-particle states localized far away from each other cannot be reorganized by a short range interaction. Interesting effects on the ground state properties require reasonably high filling factors. For the finite density system, a Fermi liq- uid approach was suggested by Imry [10] and later developed in Refs. 11.12], reducing the problem to the study of the delocalization of a few quasi-particles above the Fermi sea. From a scaling argument, one obtains that two quasi-particles need an excitation energy $\epsilon \approx t$ (irrelevant for the usual applications in solid state physics) in order to recover the enhancement factor $L_{2} / L_{1}$ of two (excited) bare particles. This quasi-particle approach may be appropriate if there is no dramatic reorganization of the ground state by the interaction. We want to consider another limit: when the Fermi liquid becomes instable as the Mott insulator is approached. To avoid any confusion, let us say that the Fermi liquid will be better named a Fermi glass in the disordered limit which we consider, characterized by Anderson localization without interaction. By Mott insulator, we mean a strongly correlated array of charges which is pinned and distorted by the random lattice.

For the TIP problem (symmetric states of two electrons with opposite spins and on-site interaction), the ratio $L_{2} / L_{1}$ is maximum [13] when the system becomes instable between two limits: free bosons for $U=0$ and hard core bosons for $U=\infty$. When $U \approx t$, the mixing of the one-particle states by the interaction is maximum and the system becomes weakly chaotic with critical spectral statistics for system sizes $M \approx L_{1}$. This critical regime cannot be described by perturbation expansions in powers of $U$ or $t^{2} / U$, starting from the free boson and hard-core boson limits respectively. Therefore, approaches starting from simple limits for the ground state (Luttinger liquid for weak disorder [14], Mott insulator for strong interaction, Fermi glass for weak interaction and large disorder) may not be valid for $U \approx t$ and large disorder, say $k_{\mathrm{f}} l \approx 1\left(k_{\mathrm{f}}^{-1}\right.$ characterizes the spacing between the electrons in the clean system and in $1 d$ the elastic mean free path is $l=L_{1}$ ).

This suggests us to study the many body ground state in the most chaotic case, between the limits which correspond to the free and hard-core particles in the archetypical TIP example. If $M$ denotes the number of sites of 
a $1 d$ lattice with $N$ spinless fermions, we expect a maximum delocalization when the parameters $(U, t, W, N$ and $M)$ are chosen in such a way that the system becomes instable between two insulating limits with different charge configurations: Anderson insulator in the Fermi limit and Mott insulator in the strongly correlated limit. The Anderson insulator yields an inhomogeneous charge configuration: strongly localized states are populated with the restriction imposed by Pauli principle. The Mott insulator yields a more or less homogeneous charge configuration. The crossover between those two limits will yield a profound spatial reorganization of the ground state, making the system more sensitive to any external perturbation as a twist in the boundary conditions or a flux $\Phi$ in a ring.

To illustrate this, we consider spinless fermions on a disordered chain with nearest neighbor (NN) interaction

$$
H=-t \sum_{i=1}^{M}\left(c_{i}^{\dagger} c_{i-1}+c_{i-1}^{\dagger} c_{i}\right)+\sum_{i=1}^{M} v_{i} n_{i}+U \sum_{i=1}^{M} n_{i} n_{i-1}
$$

and twisted boundary conditions, $c_{0}=\exp (i \Phi) c_{M}$. The operators $c_{i}\left(c_{i}^{\dagger}\right)$ destroy (create) a particle on site $i$ and $n_{i}=c_{i}^{\dagger} c_{i}$ is the occupation operator. The on-site energies $v_{i}$ are drawn from a box distribution of width $W$. Setting $t=1$, the strength of the disorder $W$ and the interaction $U$ are measured in units of the kinetic energy scale. Though strong long-range interaction always imposes an homogeneous configuration, we restrict the study to interaction between NN. This is why we begin to discuss half filling, where the ground state will be a periodic array of charges located on the even or odd sites of the chain when $U \rightarrow \infty$. This is the homogeneous configuration driven by the interaction. On the contrary, when $U \rightarrow 0$, an inhomogeneous configuration is obtained for a sufficiently strong disorder.

The numerical results are obtained with the density matrix renormalization group (DMRG) algorithm [15]. The calculation of ground state properties of interacting fermions in disordered one-dimensional systems is

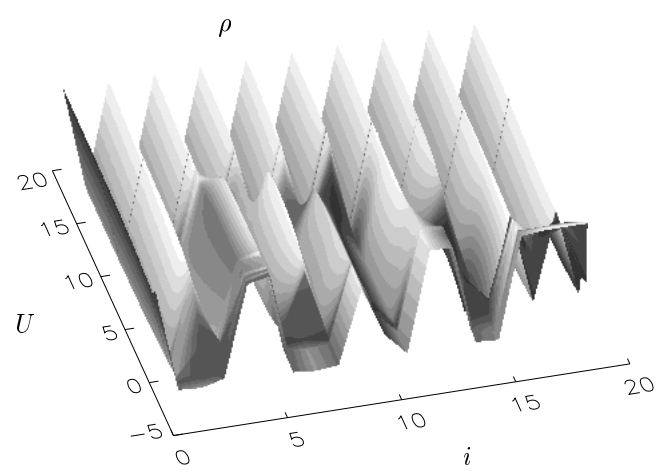

FIG. 1. Charge configuration for a typical sample (d of Fig. 2) for $N=10$ particles on $M=20$ sites at $W=9$.

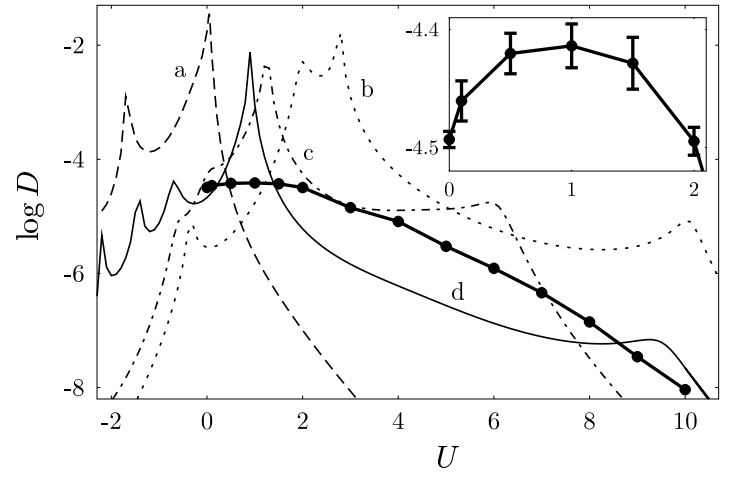

FIG. 2. Phase sensitivity $D(U)$ for four different samples with $N=10, M=20$ and $W=9$ in (decimal) logarithmic scale. $W$ and $U$ are measured in units of $t$. Thick dots and inset: average of $\log (D)$.

possible with an accuracy comparable to exact diagonalization, but for much larger systems (where we keep up to 2000 states per block 16]).

The reorganization of the ground state induced by the NN repulsion is shown in Fig. 11, where the density $\rho$ (expectation value of $n_{i}$ ) is plotted as a function of $U$ and site index $i$. To favor the inhomogeneous configuration, the disorder is taken large $(W=9)$ and $L_{1} \approx 100 / W^{2}$ is of order of the mean spacing $k_{\mathrm{f}}^{-1}=2$ between the charges. For $U \approx 0$, one can see a strongly inhomogeneous and sample dependent density, while for large $U$ a periodic array of charges sets in. These two limits are separated by a sample dependent crossover regime. For certain random configurations, the periodic array is quickly obtained by a weak repulsive interaction, while one needs a strong interaction for other samples.

To measure the delocalization effect associated to this change of configuration, we study the phase sensitivity of the ground state. The energy difference between periodic $(\Phi=0)$ and anti-periodic $(\Phi=\pi)$ boundary conditions, $\Delta E=(-)^{N}(E(0)-E(\pi))$ conveys similar information, in the localized regime, as other measures of the response of the ground state to an infinitesimal flux threading the ring: the Kohn curvature (charge stiffness) $\propto E^{\prime \prime}(\Phi=0)$ and the persistent current $J \propto-E^{\prime}(\Phi=0)$. For strictly $1 d$ systems, the sign of $E(0)-E(\pi)$ simply depends on the parity of $N$, and the factor $(-)^{N}$ makes $\Delta E$ positive.

The phase sensitivity, defined by $D(U)=(M / 2) \Delta E$, is shown in Fig. 2 for four samples at half filling with $W=9$. Both for $U \approx 0$ and $U \gg 1, D(U)$ is exponentially small, but sharp peaks appear at sample dependent values $U_{c}$, where $D\left(U_{c}\right)$ in certain samples can be 4 orders of magnitude larger than for free fermions. Remarkably, the curves for each sample do not present any singularity at $U=0$ which could allow to locate the free fermion case. Peaks can be seen at different sample dependent values of $U$ (positive or negative). Attractive interactions favor 


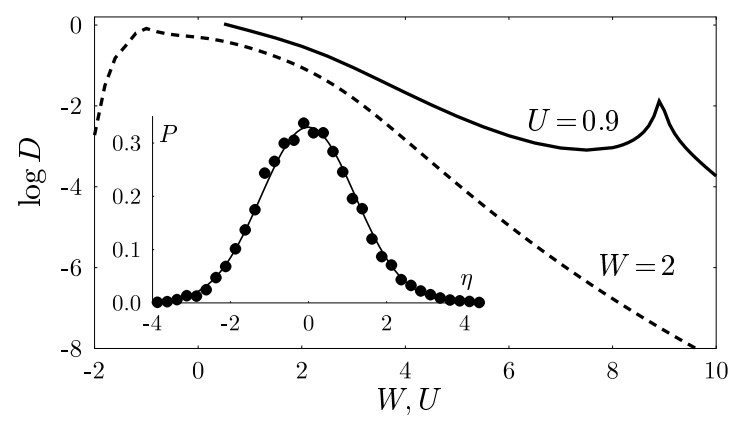

FIG. 3. Phase sensitivity for the sample d of Fig. 2. Solid: $D(W)$ at $U=0.9$; dashed: $D(U)$ at $W=2$. Inset: Probability distribution (dots) of $\eta=\log D(U=2)-\log D(U=0)$ calculated from 10000 samples $(M=20, N=10, W=9)$ fitted by a Gaussian with variance $\sigma^{2}=1.46$.

the more inhomogeneous density. Repulsive interactions favor the more homogeneous density. Free fermions correspond to an intermediate case. For small repulsive interactions, the system is an Anderson insulator slightly delocalized by $U$, and $D(U)$ increases as a function of $U$. At $U_{c}$, the regular array of charges is established, and thereafter it becomes more and more rigid (pinned by the random lattice); thus $D(U)$ decreases as a function of $U$. Certain samples go from the inhomogeneous density to the periodic array in a few steps signaled by additional peaks of sensitivity. Examining the $U$ dependence of the density of those samples, one can note local defects in the periodic array subsisting up to large values of $U$. The thresholds $U_{c}$ are strongly sample dependent giving rise to a very wide distribution of phase sensitivities: the ensemble average at a given $U$ mixes very different behaviors and provides rather misleading information. As shown in Fig. 2, $\langle\log D(U)\rangle$ decreases for repulsive interactions, except for a small interval around $U \approx t$ (inset) where a local maximum is obtained. Using 5000 samples we confirm beyond statistical uncertainty that repulsive interactions delocalize in certain parameter region. However, this small average effect is not representative of the dramatic enhancement characterizing individual samples.

We obtain approximately log-normal distributions for $D(U)$ as well as for the parameter $\eta=\log D(U=2)-$ $\log D(U=0)$ that measures the relative increase of the charge sensitivity with respect to the free fermion case. The width of the $\eta$-distribution depends on $U$, and for $U=2$ we can see that variations of $D$ over more than an order of magnitude are typical (inset of Fig. 3).

For weak disorder $\left(W=2, L_{1} \approx M\right)$ we recover the expected behavior starting from the clean limit, using bosonization and renormalization group arguments [16,14]: a repulsive interaction reinforces localization, in contrast to a (not too strong) attractive interaction which delocalizes (Fig. 3). Fixing $U=0.9$, we show (in the same graph) how one goes from the weak to the

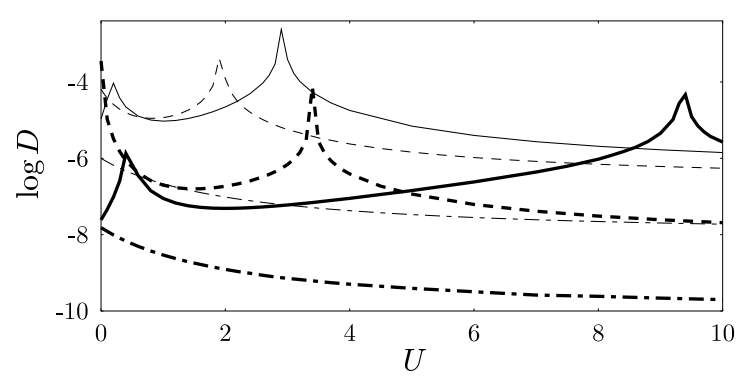

FIG. 4. Phase sensitivity for $M=40$ and $N=10$ for 3 samples with $W=4$ (thin lines) and $W=5$ (thick lines).

strong disorder limit in a given sample (the same seed of the random number generator is kept). The phase sensitivity decreases when we increase the strength of the potential fluctuations, except around $W=9\left(k_{\mathrm{f}} l \approx 1\right)$ where the charge reorganization takes place. The conclusion that a repulsive interaction favors localization is no longer valid. We underline that we have considered in this study larger disorder than in Ref. [16]. The effect is reminiscent of the Coulomb blockade phenomenon. When the occupation numbers are approximately good quantum numbers ( 0 or 1 ) transport is blocked; in the transition between such extreme configurations the occupation numbers are no longer good quantum numbers and transport is favored.

Now, we study if a repulsive interaction can delocalize at a weaker disorder for a lower filling. Of course, if we keep NN interactions, $U$ will no longer yield a fully periodic array (in contrast to a longer range interaction): the ground state can partly match the random potential without having NN electrons. One expects a more local and partial ground state reorganization than the global one discussed for half filling. In Fig. 田, we show some typical behavior obtained at quarter filling for $W=4$ and 5. For certain samples (dash-dotted), the $U=0$ Fermi glass does not have NN occupied, the charge configuration does not change when turning on the interactions, and $D(U)$ decreases with $U$. For others we can observe local charge reorganization accompanied by an increase of $D(U)$ at one (dashed) or two (solid) values of $U$. Higher disorder within the same random configuration (thicker lines) necessitates a stronger interaction $U_{c}$ to produce the charge reorganization. In our model with NN interactions and high disorder, the increase of the phase sensitivity is related to the occurrence of NN in the Fermi glass. Elementary combinatorics dictates that for a given filling factor $x=N / M$ and large $M$, the probability of obtaining a configuration without $\mathrm{NN}$ is

$$
P \simeq e^{M g(x)}, \quad g(x)=\ln \left(\frac{(1-x)^{2(1-x)}}{(1-2 x)^{(1-2 x)}}\right) .
$$

Since $g(x)<0$ in the interval $(0,1 / 2)$ of interest, configurations with $\mathrm{NN}$ occur with probability 1 in the large $M$ limit. 

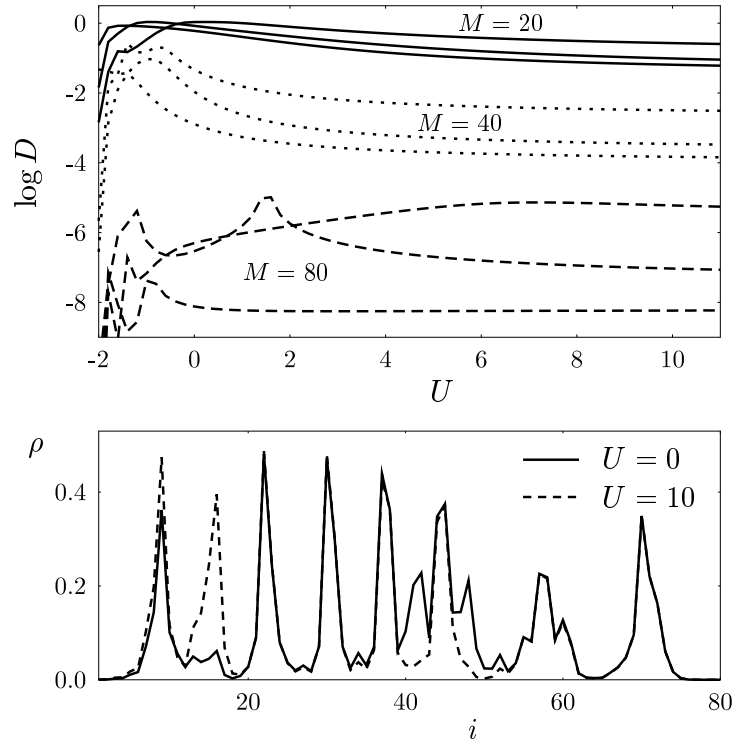

FIG. 5. Top: phase sensitivity of three samples with $W=2, N=8$ and various fillings: $M=20$ (solid), $M=40$ (dotted), and $M=80$ (dashed). Bottom: charge density at $U=0$ (solid) and $U=10$ (dashed) for the sample with $M=80$ that shows a peak around $U=1.5$.

Fig. 0 presents $D(U, x)$ at a smaller disorder $(W=2)$ for filling factors decreasing down to $1 / 10(N=8$ and $M=20,40,80)$. We underline that such a study is clearly out of reach if one is restricted to standard diagonalization algorithms. To estimate a sample dependent length $L_{1}$, one assumes that $D(U=0) \approx(M / 2) \exp \left(-2 M / L_{1}\right)$. This gives for $M=20(40) L_{1} \approx 17(10)$, corresponding to $k_{\mathrm{f}} l>1$. In this case a repulsive interaction localizes. On the other hand, for a larger size $M=80$, one recovers the delocalization effect when $D(U=0) \approx 10^{-6}$, i.e. when $L_{1} \approx 9 \approx M / N\left(k_{\mathrm{f}} l \approx 1\right)$. The peaks can be now broader and the magnitude of the effect is weaker (two orders of magnitude). Studying the charge density (bottom), we notice a slow local charge reorganization induced by a large variation of $U$. When $D(U) \approx 10^{-8}$ (one of the samples with $M=80$ ), the scale $L_{1} \approx 7$ of those samples is smaller than $M / N, k_{\mathrm{f}} l$ becomes too small and the Fermi glass cannot be reorganized by a NN interaction at this low filling.

We summarize the main conclusions that we draw from this DMRG study of one dimensional spinless fermions. (i) Each sample should be individually studied. The (log)-averages over the ensemble are not representative. (ii) The ratio $L_{1} /(M / N)$ (say $k_{\mathrm{f}} l$ ) defines different regimes for short-range repulsive interactions. For $k_{\mathrm{f}} l>1$, the interaction establishes a correlated array of charges inside $L_{1}$ which is pinned by the random lattice. The larger is $U$, the more rigid is the array, the more efficient is the pinning and the system is strongly insulating
(Mott). When $k_{\mathrm{f}} l<1$, the particles can be strongly localized far away from each other, and a short range interaction does not affect a strongly insulating ground state (Fermi glass). Only very excited quasi-particles can be delocalized by the interaction. Between those two limits, $k_{\mathrm{f}} l \approx 1$, the ground state can be deeply reorganized by a repulsive interaction $U \approx t$. This reorganization is accompanied by a large delocalization effect. Preliminary results [17] for the interaction induced charge reorganization in two dimensions lead to similar conclusions. This might be related to the problem of the metal-insulator transition [18] observed in Si-Mosfets at very low fillings. In quasi-one dimension, this might give some insight for the persistent current of a disordered multichannel ring.

We thank G. Ingold, J.-F. Joanny, I. Safi, X. Waintal and H. Weidenmüller for useful discussions. Financial support from the TMR network "Phase coherent dynamics of hybrid nanostructures" of the EU is gratefully acknowledged.

[1] "Correlated Fermions and Transport in Mesoscopic Systems", Proc. of the XXI ${ }^{s t}$ Rencontres de Moriond, ed. by T. Martin et al., Editions Frontieres (1996).

[2] L.P. Lévy et al., Phys. Rev. Lett. 64, 2074 (1990); V. Chandrasekhar et al., Phys. Rev. Lett. 67, 3578 (1991).

[3] A. Schmid, Phys. Rev. Lett. 66, 80 (1991); F. von Oppen and E. K. Riedel, ibid 84; B. L. Altshuler, Y. Gefen, and Y. Imry, ibid 88.

[4] U. Eckern, Z. Phys. B 42, 389 (1991).

[5] T. Giamarchi and B. Shastry, Phys. Rev. B 51, 10915 (1995).

[6] A. Müller-Groeling, H.A. Weidenmüller and C.H. Lewenkopf, Europhys. Lett. 22, 193 (1993).

[7] M. Abraham and R. Berkovits, Phys. Rev. Lett. 70, 1509 (1993).

[8] G. Bouzerar, D. Poilblanc, and G. Montambaux, Phys. Rev. B 49, 8258 (1994).

[9] D.L. Shepelyansky, Phys. Rev. Lett. 732607 (1994).

[10] Y. Imry, Europhys. Lett. 30405 (1995).

[11] F. von Oppen and T. Wettig, Europhys. Lett. 32, 741 (1995).

[12] Ph. Jacquod and D.L. Shepelyansky, Phys. Rev. Lett. 78, 4986 (1997).

[13] X. Waintal, D. Weinmann and J.-L. Pichard, condmat/9801134.

[14] T. Giamarchi and H. Schulz, Phys. Rev. B 37, 325 (1988).

[15] S.R. White, Phys. Rev. B 48, 10345 (1993).

[16] P. Schmitteckert et al., Phys. Rev. Lett. 80, 560 (1998).

[17] G. Benenti, X. Waintal and J.-L. Pichard, unpublished.

[18] S.V. Kravchenko et al., Phys. Rev. Lett. 774938 (1996). 